\title{
Radiation Pressure, Poynting-Robertson Drag, and Solar Wind Drag in the Restricted Three-Body Problem
}

\author{
Jer-Chyi Liou and Herbert A. Zook \\ SN3, NASA Johnson Space Center, Houston, Texas 77058 \\ E-mail: liou@sn3.jsc.nasa.gov. \\ AND \\ A. A. JACKSON \\ Lunar and Planetary Institute, Houston, Texas 77058 \\ Received December 22, 1994; revised March 21, 1995
}

In this paper, we examine the effects of radiation pressure, Poynting-Robertson (PR) drag, and solar wind drag on dust grains trapped in mean motion resonances with the Sun and Jupiter in the restricted (negligible dust mass) three-body problem. We especially examine the evolution of dust grains in the $1: 1$ resonance. As a first step, the Sun and Jupiter are idealized to both be in circular orbit about a common center of mass (circular restricted three-body problem). From the equation of motion of the dust particle in the rotating reference frame, the drag-induced time rate of change of its Jacobi "constant," $C$, is then derived and expressed in spherical coordinates. This new mathematical expression in spherical coordinates shows that $C$, in the $1: 1$ resonance, both oscillates and secularly increases with increasing time. The new expression gives rise to an easy understanding of how an orbit evolves when the radiation force and solar wind drag are included. All dust grain orbits are unstable in time when PR and solar wind drag are included in the Sun-Jupiter-dust system. Tadpole orbits evolve into horseshoe orbits; and these orbits continuously expand in size to lead to close encounters with Jupiter. Permanent trapping is impossible. Orbital evolutions of a dust grain trapped in the 1:1 resonance in the planar circular, an inclined case, an eccentric case, and the actual Sun-Jupiter case are numerically simulated and compared with each other and show grossly similar time behavior. Resonances other than $1: 1$ are also explored with the new expression. Stable exterior resonance trapping may be possible under certain conditions. One necessary condition for such a trap is derived. Trapping in interior resonances is shown to be always unstable. 1995 Academic Press, Inc.

\section{INTRODUCTION}

In a previous paper (Liou and Zook, 1995, hereinafter referred to as Paper I) we described the existence and evolution of a dust ring, of main belt asteroidal origin, composed of micrometer-sized particles that were trapped into the $1: 1$ mean motion resonance with Jupiter. It was found that a limited size range (radius $\sim 1 \mu \mathrm{m}$ ) of asteroidal dust particles, due to the radiation pressure force felt when particles are released from their parent bodies, often would be injected directly from main belt parent asteroids into the $1: 1$ mean motion resonance zone with Jupiter. There they would remain trapped for tens of thousands of years before having close encounters with Jupiter which removed them from the resonance.

When a particle is trapped, it usually starts with a tadpole-type orbit oscillating around L4 or L5. The maximum range of its libration angle increases with time. This angle gradually increases to such an extent that the particle's orbit becomes a horseshoe. The horseshoe orbit keeps expanding until it becomes possible for the particle to have a close encounter with Jupiter and, eventually, the particle is scattered out of the resonance. Radiation pressure, Poynting-Robertson (PR) drag, and solar wind drag control the orbital evolution of such particles. Otherwise, they would remain trapped in a stable state and behave just like the Trojan asteroids.

The effect of radiation pressure and PR drag at the 1:1 mean motion resonance in the restricted three-body problem has been studied to some extent previously. These studies include, for example, those of Colombo $e t$ al. (1966), Chernikov (1970), and Schuerman (1980) who discuss the positions as well as the stability of the Lagrangian equilibrium points when radiation pressure and PR drag forces are included. More recently, Murray (1994) systematically discusses the dynamical effect of general drag (nebular drag, gas drag, and PR drag) in the planar, circular, restricted three-body problem, including also a 
1 
discussion of positions and stability of the Lagrangian equilibrium points. However, these stability analyses are all based on a linear approximation that assumes the displacements of orbits from the Lagrangian equilibrium points are small and that the drag force is also small. Secure conclusions could not, from these analyses, easily be drawn about the global stability of orbits in the $1: 1$ resonance with large displacements from the Lagrangian equilibrium points (see, for example, Murray 1994. p. 480).

Earlier analyses of the time variation of the Jacobi "constant" were used, for example, to study the "Jacobi capture" of dust grains near the Earth (Colombo et al. 1966), to determine the stability of an orbit with a drag force proportional to the velocity of a particle in the rotating reference frame (e.g., Murray 1994), and the stability of Trojan precursors with nebular gas drag (Peale 1993). In Murray's paper (1994), the method is also applied, in a two-dimensional analysis, to a simple drag force proportional to the velocity of a particle in the inertial reference frame. He was not able, however, to determine (in Cartesian coordinates) the ultimate stability of the orbit at large displacements from the Lagrangian points.

Building on previous work (especially that of Murray 1994), we derive a new, and simple, mathematical expression in spherical coordinates that accurately describes how radiation pressure, PR, and solar wind drag forces change the Jacobi constant with time and thereby control the orbital evolution of dust particles in a $1: 1$ mean motion resonance location. The formula works equally well for large displacements as for small. We also apply this new expression to the orbital evolution of dust grains trapped in other types of resonances.

With this new mathematical expression, we are also better equipped than before to understand the physics behind the global evolution of particles, how their orbits expand, how their orbits change from tadpoles to horseshoes, etc. We show that $1: 1$ resonance traps are always unstable under these drag forces. Tadpole orbits will evolve into horseshoe orbits and, eventually, close encounters with Jupiter become possible and the particles exit the resonance trap. Hence, no permanent trapping is possible for dust grains in the $1: 1$ mean motion resonance. We also show that interior resonance traps are similarly unstable, but that quasi-stable exterior resonance traps may exist. One necessary condition for quasistable exterior resonance traps is derived.

The nature of the radiation force and solar wind drag is briefly summarized in Section 2 . In Section 3, we derive the time dependence of the Jacobi constant on radiation pressure, PR drag, and solar wind drag in the circular, restricted three-body problem. We also show how the time rate of change of the Jacobi constant relates to the orbital evolution of particles. The case where all three bodies are in the same plane is discussed in additional detail. In Section 4, several numerical experiments, including those of noncircular, nonplanar cases are presented. The results are compared with the theory from Section 3. The implications for micrometer-sized asteroidal dust particles and Trojan dust particles trapped in the 1:1 mean motion resonance with Jupiter is also discussed.

\section{RADIATION FORCE AND SOLAR WIND DRAG}

The orbital evolution of dust grains in the Solar System is affected by the radial solar radiation pressure force, by gravitational forces due to the Sun and planets, and by PR and solar wind drag forces. In an inertial reference frame, the equation of motion of a dust particle with geometric cross section $A$ and mass $m$ moving under the influence of the gravitational forces of the Sun, nine planets, the radiation pressure force, and the drag forces can be written as (e.g., Burns et al. 1979)

$$
\begin{aligned}
m \dot{\mathbf{v}}= & -\left[\frac{G M_{\odot} m}{r_{s}^{3}}\right] \mathbf{r}_{s}-\sum_{n=1}^{9} \frac{G M_{n} m}{r_{n}^{3}} \mathbf{r}_{n}+(\mathrm{SA} / c) Q_{\mathrm{pr}} \\
& \times\left[\left(1-(1+\mathrm{sw}) \frac{\mathbf{v} \cdot \mathbf{r}_{\mathrm{s}}}{c r_{s}}\right) \hat{\mathbf{r}}_{\mathrm{s}}-(1+\mathrm{sw}) \frac{\mathbf{v}}{\mathrm{c}}\right]
\end{aligned}
$$

where $\mathbf{v}$ is the velocity vector of the particle, $\hat{\mathbf{r}}_{\mathrm{s}}$ is its unit position vector with respect to the Sun, and $M_{\odot}$ is the Sun's mass, while $M_{n}$ and $r_{n}$ are the mass of the $n$th planet and the position vector of the particle with respect to that planet. $S, c$, and $Q_{\mathrm{pr}}$ are the solar energy flux density, the speed of light, and the radiation pressure coefficient, respectively. The velocity-independent part of the third term on the right-hand side of Eq. (1) is the radiation pressure force term, while the velocity-dependent parts are the drag terms. Solar wind drag is caused by the interaction between solar wind ions and the dust particle, and the net effect is similar to that of PR drag. The ratio of solar wind drag to PR drag is represented by sw in Eq. (1). We have assumed sw to be 0.35 (Gustafson 1994). The radiation pressure coefficient, $Q_{\mathrm{pr}}$, depends on the properties (density, shape, size, etc.) of the particle. Traditionally, a dimensionless quantity, $\beta$, is introduced to specify the effect of radiation pressure and PR drag. It is defined as

$$
\beta=\frac{\text { radiation pressure force }}{\text { solar gravitation force }}=\frac{S A Q_{p r} r_{s}^{2}}{c G M_{\odot} m}
$$

The radiation pressure force is the reason some asteroidal dust particles, with $\beta \sim 0.26$, are injected into the $1: 1$ mean motion resonance with Jupiter (see Paper I); it is responsible for changing the effective mass of the Sun as 
seen by the dust particles. Consequently, it changes the new location of the $1: 1$ mean motion resonance to $a_{n}=$ $(1-\beta)^{1 / 3} a_{\mathrm{J}}$, where $a_{\mathrm{J}}$ is the semimajor axis of Jupiter's (or any arbitrary planet's) orbit. PR and solar wind drag forces, on the other hand, cause dust particles to lose orbital energy and angular momentum and spiral toward the Sun. A detailed description of the radiation pressure force, PR, and solar wind drag forces can be found in Burns et al. (1979).

\section{THE RADIAL RADIATION PRESSURE FORCE, PR, AND SOLAR WIND DRAG IN THE RESTRICTED THREE- BODY PROBLEM}

\subsection{Time Variation of the Jacobi Constant}

In the classical circular restricted three-body problem, the three colinear Lagrangian equilibrium points, L1, L2, and L3, are unstable while the two equilateral Lagrangian equilibrium points, $\mathrm{L} 4$ and L5, are stable (with the condition that the mass ratio of the secondary to the primary is less than 0.0385); this is despite the fact that the L4 and L5 points are at effective potential maxima in the $(x$, y) plane in the rotating reference frame (e.g., Greenberg and Davis 1978). The existence of a dissipative medium may destroy the stability of the L4 and L5 regions, however, depending on the nature of the dissipative mechanism (Greenberg 1978, Yoder 1979). For dust particles in the Solar System, PR and the solar wind drag serve to take away orbital energy and angular momentum expressed in an inertial system. Their dissipative nature is well defined by the velocity-dependent terms in Eq. (1). These dissipative forces on dust grains can sometimes be counterbalanced, at least in part, by planetary gravitational perturbations, especially when the dust grain is in orbital resonance with a planet. As already achieved by others (e.g., Murray 1994), and for the convenience of the reader, we first derive the three-dimensional equations of motion of the particle in Cartesian coordinates-both in an inertial reference frame and in a reference frame that rotates with the two massive bodies. We then derive the dissipative mechanisms of the drag forces in spherical coordinates in the rotating reference frame and study their effects on the stability of the equilibrium points and on the overall evolution of an orbit.

We first define the symbols and units used in the circular, restricted three-body problem. Let $S$ and $J$ be represented by two masses $\mu_{1}$ and $\mu_{2}$, respectively (where $\mu_{1}+\mu_{2}=1, \mu_{1}>\mu_{2}$ ), moving in circular orbit around their center of mass (CM) due to mutual gravitational attraction. The inertial reference frame, $(\xi, \eta, \zeta)$, has its origin at CM. $(\xi, \eta)$ is the orbital plane of $S$ and J. The rotating reference frame $(x, y, z)$ is rotating with constant angular velocity around the collocated $\zeta$ and $z$ axes, with the $x$ axis lying along the line from CM to $J$. The distances

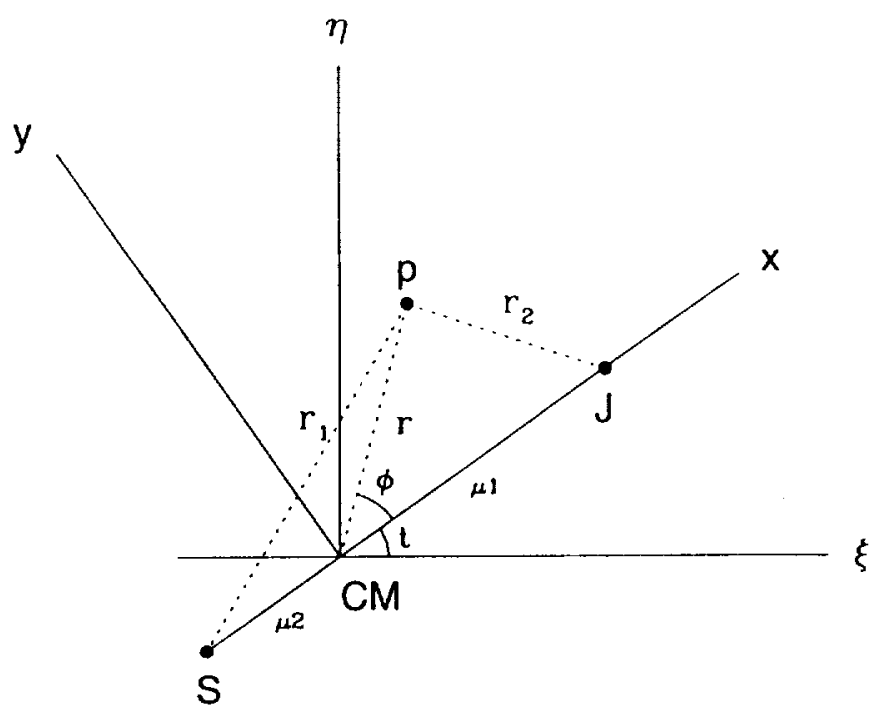

FIG. 1. The inertial coordinate system, $(\xi, \eta)$, and the coordinate system, $(x, y)$, corotating with $S$ and $J$. Both systems have their origins at the center of mass (CM) of $S$ and J. The polar coordinate system, $(r, \phi)$ is also shown. It is related to $(x, y)$ by $x=r \cos \phi$ and $y=r \sin$ $\phi$. See text for the definitions of other quantities.

from the test particle, $p$, to $\mathrm{CM}, \mathrm{S}$, and $\mathrm{J}$, are $r, r_{1}$, and $r_{2}$, respectively. The unit of length is the distance between $S$ and J. The unit of mass is the total of $S$ and $J$. The unit of time is the orbital period of $\mathrm{J}$ divided by $2 \pi$. In such normalized units, the gravitational constant and the angular velocities of $S$ and $J$ with respect to $C M$ are all unity. Figure 1 shows the relationships between these quantities in the planar case (where $\zeta$ and $\mathrm{z}$ components are zero).

The components of Eq. (1), with $\beta$ from Eq. (2), are

$$
\begin{aligned}
& \ddot{\xi}=-(1-\beta) \mu_{1} \frac{\xi-\xi_{1}}{r_{1}^{3}}-\mu_{2} \frac{\xi-\xi_{2}}{r_{2}^{3}}+(1+\mathrm{sW}) F_{\mathrm{PR}, \zeta} \\
& \ddot{\eta}=-(1-\beta) \mu_{1} \frac{\eta-\eta_{1}}{r_{1}^{3}}-\mu_{2} \frac{\eta-\eta_{2}}{r_{2}^{3}}+(1+\mathrm{sw}) F_{\mathrm{PR}, \eta} \\
& \ddot{\zeta}=-(1-\beta) \mu_{1} \frac{\zeta-\zeta_{1}}{r_{1}^{3}}-\mu_{2} \frac{\zeta-\zeta_{2}}{r_{2}^{3}}+(1+\mathrm{sw}) F_{\mathrm{PR}, \zeta} .
\end{aligned}
$$

These are the components of the equation of motion of a dust grain in an inertial reference frame with solar radiation pressure and PR and solar wind drag terms. Here the radiation pressure force has been combined into the first terms on the right-hand side of Eq. (3) and the drag components are

$$
\begin{aligned}
& F_{\mathrm{PR}, \xi}= \\
& -\beta \frac{\mu_{1}}{c r_{1}^{2}}\left\{\frac{\left[\left(\xi-\xi_{1}\right) \dot{\xi}+\left(\eta-\eta_{1}\right) \dot{\eta}+\left(\zeta-\zeta_{1}\right) \dot{\zeta}\right]\left(\xi-\xi_{1}\right)}{r_{1}^{2}}+\dot{\xi}\right\}
\end{aligned}
$$




$$
\begin{aligned}
& F_{\mathrm{PR}, \eta}= \\
& -\beta \frac{\mu_{1}}{c r_{1}^{2}}\left\{\frac{\left[\left(\xi-\xi_{1}\right) \dot{\xi}+\left(\eta-\eta_{1}\right) \dot{\eta}+\left(\zeta-\zeta_{1}\right) \dot{\zeta}\right]\left(\eta-\eta_{1}\right)}{r_{1}^{2}}+\dot{\eta}\right\} \\
& F_{\mathrm{PR}, \zeta}= \\
& -\beta \frac{\mu_{1}}{c r_{1}^{2}}\left\{\frac{\left[\left(\xi-\xi_{1}\right) \dot{\xi}+\left(\eta-\eta_{1}\right) \dot{\eta}+\left(\zeta-\zeta_{1}\right) \dot{\zeta}\right]\left(\zeta-\zeta_{1}\right)}{r_{1}^{2}}+\dot{\zeta}\right\} .
\end{aligned}
$$

Using the coordinate transformation,

$$
\left\{\begin{array}{l}
\xi=x \cos t-y \sin t \\
\eta=x \sin t+y \cos t
\end{array}\right.
$$

Equation (3) becomes, in the rotating reference frame,

$$
\begin{aligned}
\ddot{x}-2 \dot{y}= & x-\frac{(1-\beta) \mu_{1}}{r_{1}^{3}}\left(x+\mu_{2}\right) \\
& -\frac{\mu_{2}}{r_{2}^{3}}\left(x-\mu_{1}\right)+(1+\mathrm{sw}) F_{\mathrm{PR}, x} \\
\ddot{y}+2 \dot{x}= & y-\frac{(1-\beta) \mu_{1}}{r_{1}^{3}} y-\frac{\mu_{2}}{r_{2}^{3}} y+(1+\mathrm{sw}) F_{\mathrm{PR}, y} \\
\ddot{z}= & -\frac{(1-\beta) \mu_{1}}{r_{1}^{3}} z-\frac{\mu_{2}}{r_{2}^{3}} z+(1+\mathrm{sw}) F_{\mathrm{PR}, z}
\end{aligned}
$$

or

$$
\begin{aligned}
\ddot{x}-2 \dot{y} & =\frac{\partial U}{\partial x}+(1+\mathrm{sw}) F_{\mathrm{PR}, x} \\
\ddot{y}+2 \dot{x} & =\frac{\partial U}{\partial y}+(1+\mathrm{sw}) F_{\mathrm{PR}, y} \\
\ddot{z} & =\frac{\partial U}{\partial z}+(1+\mathrm{sw}) F_{\mathrm{PR}, z},
\end{aligned}
$$

where

$$
U \equiv \frac{(1-\beta) \mu_{1}}{r_{1}}+\frac{\mu_{2}}{r_{2}}+\frac{1}{2}\left(x^{2}+y^{2}\right)
$$

is the modified potential in the rotating reference frame. The PR drag components in $(x, y, z)$ coordinate system are

$$
\begin{aligned}
F_{\mathrm{PR}, x}= & -\beta \frac{\mu_{1}}{c r_{1}^{2}}\left\{\frac{\left[\left(x+\mu_{2}\right) \dot{x}+\left(\dot{y}-\mu_{2}\right) y+\dot{z} z\right]\left(x+\mu_{2}\right)}{r_{1}^{2}}\right. \\
& +(\dot{x}-y)\}
\end{aligned}
$$

$$
\begin{aligned}
& F_{\mathrm{PR}, y}=-\beta \frac{\mu_{1}}{c r_{1}^{2}}\left\{\frac{\left[\left(x+\mu_{2}\right) \dot{x}+\left(\dot{y}-\mu_{2}\right) y+\dot{z} z\right] y}{r_{1}^{2}}+(\dot{y}+x)\right\} \\
& F_{\mathrm{PR}, z}=-\beta \frac{\mu_{1}}{c r_{1}^{2}}\left\{\frac{\left[\left(x+\mu_{2}\right) \dot{x}+\left(\dot{y}-\mu_{2}\right) y+\dot{z} z\right] z}{r_{1}^{2}}+\dot{z}\right\} .
\end{aligned}
$$

When there are no dissipative forces (i.e., $F_{\mathrm{PR}, i}=0$ ), there is a constant of the motion (e.g., Moulton 1914), the Jacobi constant, defined as

$$
C \equiv 2 U-\left(\dot{x}^{2}+\dot{y}^{2}+\dot{z}^{2}\right) \text {. }
$$

If we multiply the first of Eq. (6) by $\dot{x}$, the second of Eq. (6) by $\dot{y}$, and the third of Eq. (6) by $\dot{z}$, and add, and use Eq. (10), we obtain the time variation of the Jacobi constant due to drag forces,

$$
\begin{aligned}
\dot{C}= & 2(1+\mathrm{sw}) \frac{\beta \mu_{1}}{c r_{1}^{2}}\left[\left(\dot{x}^{2}+\dot{y}^{2}+\dot{z}^{2}\right)-(y \dot{x}-x \dot{y})\right. \\
& \left.+\frac{\left(x \dot{x}+y \dot{y}+z \dot{z}+\mu_{2} \dot{x}\right)\left(x \dot{x}+y \dot{y}+z \dot{z}+\mu_{2} \dot{x}-\mu_{2} y\right)}{r_{1}^{2}}\right]
\end{aligned}
$$

or

$$
\begin{aligned}
\dot{C}= & 2(1+\mathrm{sw}) \frac{\beta \mu_{1}}{c r_{1}^{2}}\left[\left(\dot{x}^{2}+\dot{y}^{2}+\dot{z}^{2}\right)-(y \dot{x}-x \dot{y})\right. \\
& \left.+\frac{(x \dot{x}+y \dot{y}+z \dot{z})^{2}}{r_{1}^{2}}+O\left(\mu_{2}\right)\right] .
\end{aligned}
$$

Here $O\left(\mu_{2}\right)$ are terms which are multiplied by the secondary mass, $\mu_{2}$, and in the case of the Sun-Jupiter system where $\mu_{2} \sim 0.001$, these terms can be neglected. This formula is similar, in part (the major difference being the $r_{1}^{-2}$ dependence of PR and solar wind drag that we use), to that derived in two dimensions by Murray (1994). It will prove to be much easier, as we shall see, to visualize particle dynamics, and the behavior of $C$, in a spherical coordinate system $(r, \theta, \phi)$ given by the transformation,

$$
\left\{\begin{array}{l}
x=r \cos \theta \cos \phi \\
y=r \cos \theta \sin \phi \\
z=r \sin \theta .
\end{array}\right.
$$

Here $r$ is the radial distance from CM to the particle, $\theta$ is the latitude angle measured from $(x, y)$ plane toward positive $\mathrm{z}$ direction, and $\phi$ is the longitude angle measured counterclockwise from positive $x$ direction toward positive $y$ direction. With this transformation, Eq. (12) becomes 


$$
\begin{aligned}
\dot{C}= & 2(1+\mathrm{sw}) \frac{\beta \mu_{1}}{c r_{1}^{2}}\left[\dot{r}^{2}+(r \dot{\theta})^{2}+(r \dot{\phi} \cos \theta)^{2}\right. \\
& \left.+r^{2} \dot{\phi} \cos ^{2} \theta+\left(\frac{r \dot{r}}{r_{1}}\right)^{2}\right] .
\end{aligned}
$$

If we now use the law of cosines, $\left(r / r_{1}\right)^{2}$ can be expressed as

$$
\left(\frac{r}{r_{1}}\right)^{2} \equiv 1-\frac{2 \mu_{2} r \cos \psi}{r_{1}^{2}}-\left(\frac{\mu_{2}}{r_{1}}\right)^{2}=1+O\left(\mu_{2}\right)
$$

where $\psi$ is the angle between the positive $x$ axis and the position vector of the particle. This angle becomes $\phi$ when $\theta$ equals to zero. In the Sun-Jupiter-dust system, $\mu_{2} \sim 0.001, r_{1} \sim r$, and Eq. (14) can be written as

$$
\begin{aligned}
\dot{C}= & 2(1+\mathrm{sw}) \frac{\beta \mu_{1}}{c}\left[2\left(\frac{\dot{r}}{r}\right)^{2}+\dot{\theta}^{2}\right. \\
& \left.+(\dot{\phi} \cos \theta)^{2}+\dot{\phi} \cos ^{2} \theta\right] .
\end{aligned}
$$

This equation shows that when PR and solar wind drag are included in the circular Sun-Jupiter-dust system, $C$ has three positive-definite terms and one term depending on $\dot{\phi}$ (the last term), which depends on the location of the resonance. In a 1:1 resonance, the sign of $\dot{\phi}$ oscillates in time and its magnitude is less than unity. In an interior resonance $\dot{\phi}$ is positive-definite while in an exterior resonance, $\dot{\phi}$ is negative-definite. As $C$ (see Eq. (10)) defines the locus of coordinates where velocity is zero, an increasing or decreasing $C$ implies increasing or decreasing exclusion zones. This expression is independent of how far an orbit is from any given Lagrangian equilibrium point. It can be used to find the stability of an orbit in a particular type of resonance. Detailed discussions of this expression in various types of resonances are in Sections 3.3 and 3.5.

In the classical circular restricted three-body problem, the $C$ values associated with five Lagrangian equilibrium points characterize certain stable orbits that a test particle could have. Except for $C$ values at the L4 and LS points, the $C$ values define the "forbidden regions" (where the velocity of the test particle would be imaginary). Forbidden regions and regions where motion is possible are separated by zero velocity surfaces or, in the planar case, by zero velocity curves (ZVCs). For example, a test particle with a near circular orbit with a $C$ value between $C_{\mathrm{L} 3}$ and $C_{\mathrm{L} A}$ has a tadpole-type orbit. Such a particle can never enter a certain region around $\mathrm{L} 4$ (or $\mathrm{L} 5$, depending on where the particle is placed initially). Here $C_{\mathrm{LN}}$ is the Jacobi constant associated with the zero velocity surface that contains the Lagrangian point $\mathrm{LN}$. A test particle with a near circular orbit with a $C$ value between $C_{\mathrm{L} 2}$ and $C_{\mathrm{L} 3}$ has a horseshoe-type orbit (e.g., Szebehely 1967, Danby 1989).

In the classical circular restricted three-body problem, $C$ is a constant. A test particle in a $1: 1$ resonance cannot change its orbit from a tadpole to a horseshoe or vice versa. This is no longer true when the drag forces are included. The radiation pressure force reduces the effective mass of the Sun and changes the locations of the Lagrangian equilibrium points. The drag forces also have some effect on the locations of the Lagrangian equilibrium points as indicated by Eqs. (6) and (9).

It has been shown previously, in the two-dimensional case, that, in a $1: 1$ resonance with PR drag, a test particle with a small initial displacement from L4 (or L5) will oscillate around that equilibrium point, with the displacement growing exponentially in time (e.g., Colombo et al. 1966, Schuerman 1980, Murray 1994). However, when the displacement becomes large, their linear approximation cannot be used and firm conclusions could not easily be drawn about the evolution of an orbit. Our new way of using the Jacobi constant, however, has no such limitation, if the variation in $C$ can be mathematically expressed as is the case with Eq. (16). Together with Eq. (10), they can be used to determine the time dependence of the global behavior of an orbit regardless of how far the orbit is from any one given Lagrangian equilibrium point.

In the Solar System, most of the asteroids are concentrated toward the ecliptic. This is also true for the micrometer-sized asteroidal dust particles that become trapped in the 1:1 mean motion resonance with Jupiter, where their inclinations are less than $20^{\circ}$ (Paper I). In such a case, a coplanar circular restricted Sun-Jupiter-dust formulation is a good first approximation and provides a good foundation for understanding dust motion in the actual Sun-Jupiter system. Likewise for many dust grains originating from Trojan asteroids. In such a case, Eq. (16) can be simplified to

$$
\dot{C}=2(1+\mathrm{sw}) \frac{\beta \mu_{1}}{c}\left[2\left(\frac{\dot{r}}{r}\right)^{2}+\dot{\phi}^{2}+\dot{\phi}\right] .
$$

\subsection{Locations of the Lagrangian Equilibrium Points and the Zero-Velocity Curves}

In order to calculate the locations of the Lagrangian equilibrium points, Eq. (6) must be solved with the condition that all time derivatives are zero. The equations become

$$
\begin{aligned}
0= & x-\frac{(1-\beta) \mu_{1}}{r_{1}^{3}}\left(x+\mu_{2}\right)-\frac{\mu_{2}}{r_{2}^{3}}\left(x-\mu_{1}\right) \\
& +\frac{(1+s w) \beta \mu_{1}}{r_{1}^{2}}\left[\frac{\mu_{2} y\left(x+\mu_{2}\right)}{c r_{1}^{2}}+\frac{y}{c}\right]
\end{aligned}
$$




$$
\begin{aligned}
0= & y\left[1-\frac{(1-\beta) \mu_{1}}{r_{1}^{3}}-\frac{\mu_{2}}{r_{2}^{3}}\right]+\frac{(1+\mathrm{sw}) \beta \mu_{1}}{r_{1}^{2}} \\
& \times\left[\frac{\mu_{2} y^{2}}{c r_{1}^{2}}-\frac{x}{c}\right] \\
0= & z\left[\frac{(1-\beta) \mu_{1}}{r_{1}^{3}}+\frac{\mu_{2}}{r_{2}^{3}}-\frac{(1+s w) \beta \mu_{1} \mu_{2} y}{c r_{1}^{4}}\right] .
\end{aligned}
$$

There are two possible solutions in Eq. (20),

$$
\frac{(1-\beta) \mu_{1}}{r_{1}^{3}}+\frac{\mu_{2}}{r_{2}^{3}}-\frac{(1+s w) \beta \mu_{1} \mu_{2} y}{c r_{1}^{4}}=0
$$

or

$$
z=0
$$

The first solution corresponds to equilibrium points outside the $(x, y)$ plane while the second solution corresponds to equilibrium points in the $(x, y)$ plane. We examine first the out of the $(x, y)$ plane possibility. It is obvious from Eq. (21) that if $\beta$ is no larger than 1 , the only possible solution is to have $y>0$. If we substitute Eq. (21) into Eq. (19), we get

$$
0=1-\frac{(1+s w) \beta \mu_{1} x}{c r_{1}^{2} y}
$$

With the condition that $y>0$, the only possible solution is to have $x>0$.

If we now divide both sides of Eq. (18) by $\left(x+\mu_{2}\right)$ and again use Eq. (21), we get

$$
0=\frac{x}{x+\mu_{2}}+\frac{\mu_{2}}{r_{2}^{3}}\left[\frac{1}{x+\mu_{2}}\right]+\frac{(1+s w) \beta \mu_{1} y}{c r_{1}^{2}\left(x+\mu_{2}\right)}
$$

With the conditions that $x>0$ and $y>0$, the right-hand side of Eq. (24) can never equal to 0 . Therefore, we can conclude that, when the radiation pressure force on a particle is smaller than the gravitational attraction from the Sun, there are no equilibrium points outside the $(x, y)$ plane. This is also easy to show geometrically.

For the $z=0$ case, Eqs. (18) and (19) can be solved using Taylor series expansions in $r_{1}$ and $r_{2}$ and some approximations (e.g., Schuerman 1980, Murray 1994) or, more directly, can be solved numerically. The latter method is used here. We show the locations of the five Lagrangian equilibrium points for different $\beta$ values in

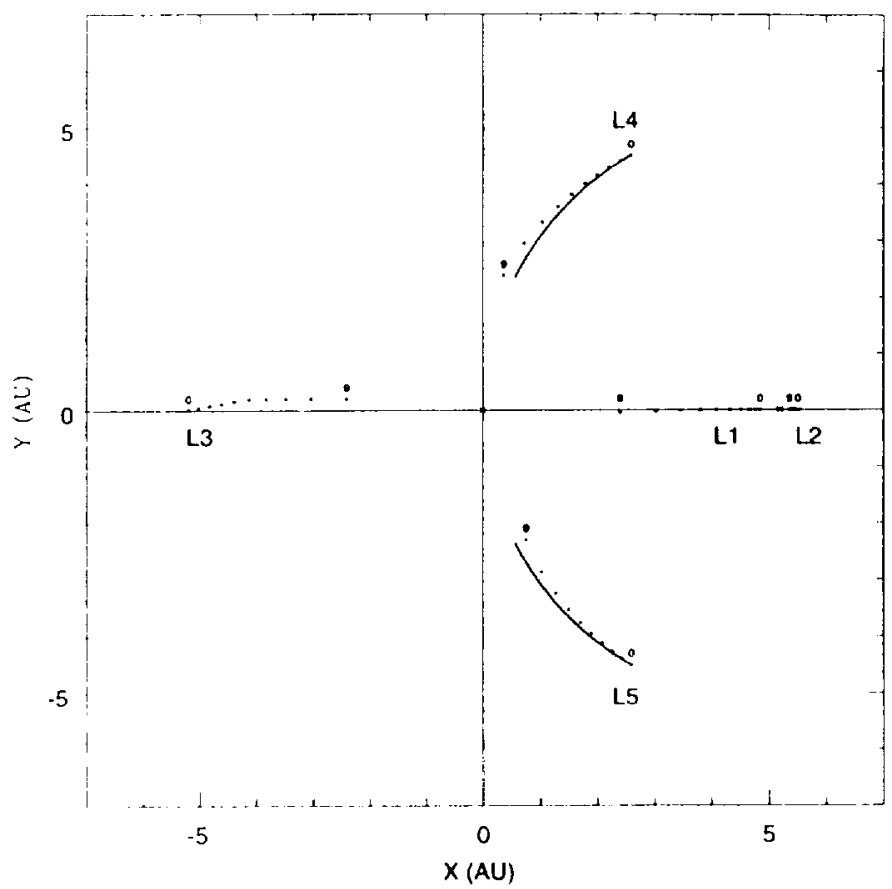

FIG. 2. The locations of the five Lagrangian equilibrium points for different $\beta$ values (sw is always 0.35 in our calculation). They are presented by black dots. $A$ " 0 " indicates the position when $\beta=0$ while a " 9 " indicates the position when $\beta=0.9$. The dots in between " 0 " and "9" are each different by 0.1 in $\beta$ value. The positions of the Sun and Jupiter are indicated by crosses. Two thick curves indicate the paths of $L 4$ (that in the first quadrant) and L5 (that in the fourth quadrant) from $\beta=0$ to $\beta=0.9$ if the effect of drag is ignored; this is the same curve as shown by Schuerman (1980) as part of an arc of a circle of unit radius centered on the secondary mass (Jupiter).

Fig. 2. It can be seen that the radiation pressure force has the most effect on changing the equilibrium point locations.

The Jacobi constant associated with the zero-velocity curve that contains a given Lagrangian equilibrium point can be obtained using Eqs. (8) and (10). The results for $\beta=0.26$ (or radius $\sim 1 \mu \mathrm{m}$ ), sw $=0.35$ particles in a circular Sun-Jupiter-dust system are listed in Table I.

All the Jacobi constants in Table I are less than 3 . This

TABLE I

Locations of the Lagrangian Equilibrium Points and the Jacobi Constants Associated with the ZVCs That Contain those Points

\begin{tabular}{lrcc}
\hline & $X(\mathrm{AU})$ & $Y(\mathrm{AU})$ & \multicolumn{1}{c}{$C_{\mathrm{LN}}$} \\
\hline L1 & 4.5825 & $-1.2 \mathrm{E}-4$ & 2.4688134 \\
L2 & 5.4560 & 0.0 & 2.5468315 \\
L3 & -4.7047 & $9.64 \mathrm{E}-2$ & 2.4555497 \\
L4 & 2.0866 & 4.2128 & 2.4539510 \\
L5 & 2.1561 & -4.1776 & 2.4539510 \\
\hline
\end{tabular}

Note. $\beta=0.26$ and sw $=0.35$, in the circular Sun-Jupiter-dust system (the semimajor axis of Jupiter is set to $5.2 \mathrm{AU}$ ). 


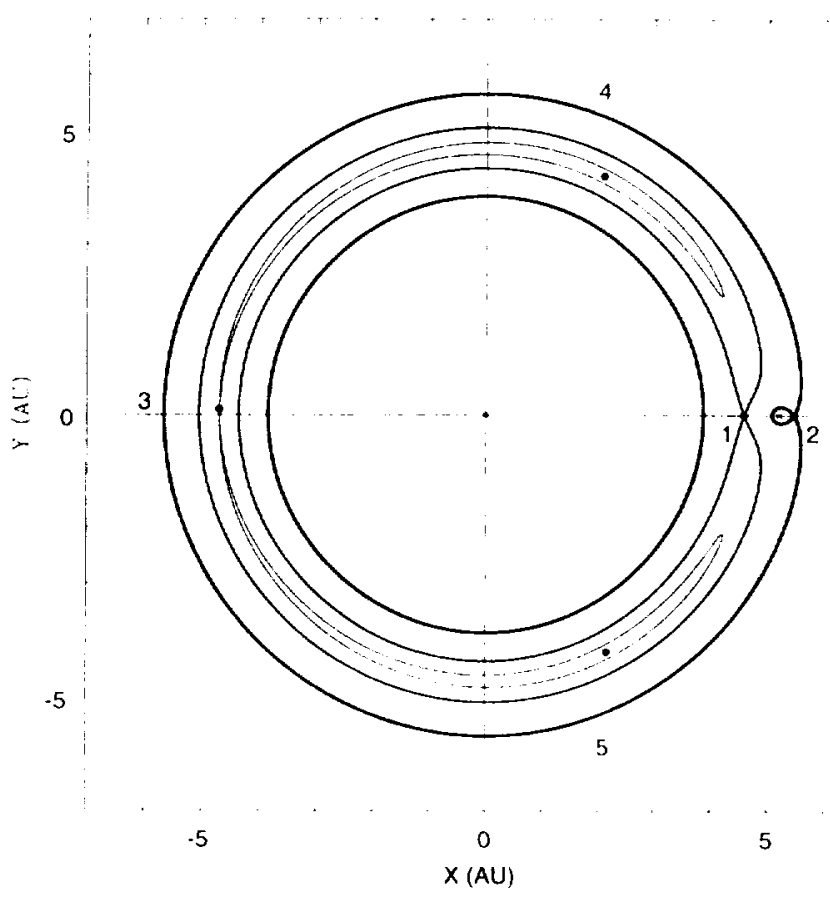

FIG. 3. Five Lagrangian equilibrium points and three critical ZVCs of the Sun-Jupiter-dust system with $\beta=0.26$, sw $=0.35$. The positions of the Sun and Jupiter are indicated by crosses. While LI (between Jupiter and Sun) and L2 (outside Jupiter, along $+x$ direction) are along the $x$ axis, L.3 has a noticeable nonzero $y$ component. The thin, intermediate, and thick curves are the $\mathrm{ZVCs}$ that contain $\mathrm{L} 3, \mathrm{L1}$, and $\mathrm{L} 2$, respectively.

is due to the effect of radiation pressure. The positions of five Lagrangian equilibrium points and the ZVCs that pass through $\mathrm{L} 1, \mathrm{~L} 2$, and $\mathrm{L} 3$ points in the $(x, y)$ plane are shown in Fig. 3. Apparently the radiation force does not change the general shapes of these ZVCs. This can be seen by comparing Fig. 3 with the classical ZVCs (e.g., Szebehely 1967). However, there are two major differences. The first one, as pointed out by Colombo et al. (1966) and more clearly demonstrated by Murray (1994) and depending on the nature and strength of the drag force, is that the locations of $\mathrm{L} 1, \mathrm{~L} 2$, and $\mathrm{L} 3$ may have nonzero $y$ components. With PR and solar wind drag, the existence of nonzero $y$ components for $\mathrm{L} 1, \mathrm{~L} 2$, and $\mathrm{L} 3$ can be verified easily from Eq. (19). However, only the $y$ components of $\mathrm{L} 3$ and $\mathrm{L} 1$ (with very large $\beta$ ) are noticeable in Fig. 2. The second difference for our case of $\beta=$ 0.26 , contrary to the classical $\beta=0$ case, is that $C_{\mathrm{L}}$ is smaller than $C_{\mathrm{L} 2}$. Consequently, as $C$ increases from $C_{\mathrm{L} 3}$, the corresponding horseshoe orbit expands and evolves into either a prograde orbit inside an inner oval (containing $S$ ), or a retrograde orbit outside an outer oval (contain $J$ ) when the $C$ value reaches $C_{\mathrm{L} 1}$. As $C$ further increases, the ZVC eventually divides the plane into three possible regions for an orbit (when $C$ reaches $C_{\mathrm{L} 2}$ ). They are a prograde orbit inside a small oval around $\mathrm{S}$, an orbit inside a smaller oval around $J$, and a retrograde orbit outside the S-J system. Here the "prograde" and "retrograde" orbits all refer to the rotating reference frame.

\subsection{Evolution of an Orbit in a 1:1 Resonance and the Effect of Inclination and Eccentricity}

For a particle trapped in a $1: 1$ mean motion resonance in the planar case, the sign of $\dot{\phi}$ oscillates with time and its magnitude is less than unity. This means that $C$ varies periodically with $\dot{\phi}$ and secularly increases with time due to the $\dot{\phi}^{2}$ and $(\dot{r} / r)^{2}$ terms (see Eq. (17)). The $\dot{\phi}^{2}$ is the dominant secular term for a low eccentricity orbit. This secular increase in $C$ leads to the expansion of the forbidden region where particle motion is imaginary. This gradually forces the tadpole or horseshoe orbits to expand. For example, if a particle starts in near circular orbit with a small displacement ("small" is not a necessary condition) from $\mathrm{L} 4$ in the rotating reference frame, its corresponding $C$ value will be slightly larger than $C_{\mathrm{L} 4}$. The forbidden region then, which contains $L 4$, is small. The particle moves clockwise around, and exterior to the tadpoletype forbidden region. As the particle moves around the forbidden region, $C$ undergoes a sinusoidal variation due to $\dot{\phi}$ and a smaller secular increase due primarily to $\dot{\phi}^{2}$. This increase in $C$ causes the forbidden region to expand. This, in turn, forces the particle's orbit to expand. When $C$ reaches $C_{\mathrm{L} 3}$, the forbidden region becomes a horseshoe, and the particle must orbit outside that horseshoe. As $C$ further increases, the horseshoe orbit expands to such an extent that close encounters with $\mathrm{J}$ become possible. Eventually, a close encounter with $J$ will remove the particle from the resonance. Thus, all orbits are unstable. No permanent trapping in the $1: 1$ mean motion resonance is possible unless a particle is put right at a given Lagrangian equilibrium point, which is highly unlikely in the real Solar System (Paper I).

In the nonplanar case, where the orbit of the dust particle is not in the orbital plane of the planet, the conclusion that all orbits are unstable is still true as shown by Eq. (16). In this case, it is still the expansion of the zerovelocity surface that forces the orbit to expand. However, because of the effect of $\theta$ on both $\dot{\phi}$ and $\dot{\phi}^{2}$, and the additional term of $\dot{\theta}^{2}$, the short-term variation as well as the secular increase components will be somewhat different from those of the planar case. In addition to the projection effect of $\dot{\phi}$ on the $(x, y)$ plane, the amplitude of the short-term variation will be smaller by a factor of $\cos ^{2} \theta$. The net result depends on both the inclination and the orientation of the orbit (i.e., where the longitude of the ascending node is).

In the classical elliptic restricted three-body problem where the planet is in an eccentric orbit and where no 
radiation force is involved, the Jacobi integral is no longer a constant of the motion. The Jacobi constant becomes a function of the eccentricity of the planet and time (e.g., Szebehely 1967, Marchal 1990). The positions of the Lagrangian equilibrium points also vary. Here we limit ourselves to a simple qualitative discussion on the effect of eccentricities of dust grain and of Jupiter's orbits. When the eccentricities of both Jupiter and the dust particle are included in the system, additional time-varying periodic factors will be combined with $\dot{r}, \dot{\phi}$, and $\dot{\theta}$ in Eq. (16) to modify the problem. This does not change the general conclusion that $C$ has a large short-term variation and small secular increase components. All orbits are unstable under PR and solar wind drag. However, because of the strong effect of the test particle's eccentricity on its $\dot{r}$, the overall evolution will be sped up and the speed up rate increases as the test particle's eccentricity increases.

All the above analytical results are verified in our numerical simulations in Section 4.

\subsection{Damping of the Inclination}

PR drag is an in-plane drag force and has no effect on the inclination of an orbit in a Sun-dust two-body system (e.g., Burns et al. 1979). However, in a Sun-planet-dust three-body system, the inclination of the dust grain can be changed. This is especially obvious when the dust grain is in a 1:1 mean motion resonance with a planet. If we substitute $F_{\mathrm{PR}, z}$ from Eq. (9) into the $\ddot{z}$ equation in Eq. (6), we have

$$
\begin{aligned}
\ddot{z}= & -\left[\frac{(1-\beta) \mu_{1}}{r_{1}^{3}}+\frac{\mu_{2}}{r_{2}^{3}}+(1+\mathrm{sw}) \frac{\beta \mu_{1}(r \dot{r})}{c r_{1}^{4}}\right] z \\
& -\left[(1+\mathrm{sw}) \frac{\beta \mu_{1}}{c r_{1}^{2}}\right] \dot{z},
\end{aligned}
$$

where the $\mu_{2}$ terms in $F_{\mathrm{PR}, z}$ have been dropped. This equation describes a typical "underdamped" oscillator (e.g., Marion 1970), with an $e$-folding time

$$
t_{e}=\frac{2 c r_{1}^{2}}{(1+\mathrm{sw}) \beta \mu_{1}},
$$

where all quantities are in the normalized units. In a $1: 1$ mean motion resonance, the damping in the amplitude of the $z$ motion leads to the damping in the inclination of the dust grain. The $e$-folding time here is much longer than the PR drag life time of a dust grain, if it is not trapped in any resonance.

\subsection{Applications to Interior and Exterior Resonances}

Our result (Eq. (16)) can be applied to study interior and exterior resonances as well. For a dust grain to drift toward and become trapped into an interior resonance with a planet, although it has been argued analytically, using Hamiltonian mechanics, to be impossible (e.g., Henrard and Lemaitre 1983, Peale 1986), such trapping has actually been observed in numerical simulations (Jackson and Zook 1992, Liou 1993, Marzari and Vanzani 1994). In all numerical simulations, the trapping is found to be only temporary. Expression Eq. (16) does not predict whether or not trapping into interior resonances is possible. However, it does show that trapping intervals in interior resonances are always only temporary.

If a dust particle does get trapped in an interior resonance with a planet, its orbital angular velocity is larger than that of the planet. Thus, in the rotating reference frame, the particle's $\dot{\phi}$ is positive all the time. This implies all terms on the right-hand side of Eq. (16) are positive. As $C$ increases, without limit, the dust grain is eventually forced to leave the resonance and continue its journey toward the Sun. Permanent trapping will only be possible if a case can be found where $\dot{C}$. on average, is zero.

In an exterior resonance, the particle's $\dot{\phi}$, in the rotating reference frame, is negative all the time. Therefore, in order to reach a possible equilibrium trapping, $(\dot{r} / r)^{2}$ and $/$ or $\dot{\theta}^{2}$ and $(\dot{\phi} \cos \theta)^{2}$ must just be large enough to counterbalance the effect of $\dot{\phi}$ and lead to $\dot{C}=0$. This implies that in order to reach an equilibrium exterior trap, the eccentricity and/or inclination of the dust grain must reach certain values. Planetary resonant perturbations (e.g., Weidenschilling and Jackson 1993) then are required to keep the orbital eccentricity at the critical value of $e$ for $\dot{C}$ to equal zero, in the planar case.

Another way of treating this problem is to look at the variation of $C$ in orbital parameter space. In the planar case,

$$
\begin{aligned}
\frac{d C}{d t}= & {\left[\frac{\partial C}{\partial a}\left(\frac{d a}{d t}\right)_{\mathrm{PR}}+\frac{\partial C}{\partial e}\left(\frac{d e}{d t}\right)_{\mathrm{PR}}\right] } \\
& +\left[\frac{\partial C}{\partial a}\left(\frac{d a}{d t}\right)_{\text {grav. }}+\frac{\partial C}{\partial e}\left(\frac{d e}{d t}\right)_{\text {grav }}\right] .
\end{aligned}
$$

The second bracket on the right-hand side is zero and the rates of change in $a$ and $e$ due to PR drag are (Wyatt and Whipple 1950)

$$
\left(\frac{d a}{d t}\right)_{\mathrm{PR}}=\frac{-\beta G M_{\odot}}{c} \frac{\left(2+3 e^{2}\right)}{a\left(1-e^{2}\right)^{3 / 2}}
$$

and

$$
\left(\frac{d e}{d t}\right)_{\mathrm{PR}}=\frac{-\beta G M_{\odot}}{c} \frac{5 e}{2 a^{2}\left(1-e^{2}\right)^{\mathrm{I} / 2}} .
$$


The expression of $C$ in terms of the orbital elements of an orbit is the Tisserand criterion (e.g., Moulton 1914)

$$
C=\frac{(1-\beta)}{a}+2 \sqrt{(1-\beta) a\left(1-e^{2}\right)},
$$

where $a$ and $e$ are the dust grain's semimajor axis and eccentricity, respectively. This expression is valid when the planet is in circular orbit and when the dust grain is not too close to the planet. Even though the dust grain can come close to the planet during part of its revolution, this expression is still good in describing the general behavior of the dust grain's orbit. If we calculate the partial derivatives of $C$ with $a$, and $e$, using Eq. (30) and substitute the results, together with Eqs. (28) and (29), into Eq. (27), the condition for $\dot{C}=0$ is

$$
\left[\frac{a^{3}}{1-\beta}\right]^{1 / 2}=\frac{2+3 e^{2}}{2\left(1-e^{2}\right)^{3 / 2}}
$$

When a dust grain is in a $(p+q) / p$ exterior mean motion resonance with a planet,

$$
\left[\frac{a^{3}}{1-\beta}\right]^{1 / 2}=\frac{p+q}{p}
$$

where $p$ and $q$ are positive integers (see also Weidenschilling and Jackson 1993). This leads to

$$
\frac{2+3 e^{2}}{2\left(1-e^{2}\right)^{3 / 2}}=\frac{p+q}{p}
$$

This means, for a given $(p+q) / p$ exterior mean motion resonance, there exists an eccentricity (of the dust grain) according to Eq. (33) that ensures $\dot{C}=0$. It is not yet clear whether completely stable external resonances actually exist, but $\dot{C}=0$ is a necessary condition if such stable resonances exist. We have noted that in long-lived threebody resonances, the eccentricity of the dust grain drifts close to the value of $e$ obtained from Eq. (33). In fact the $e$ obtained from Eq. (33) seems to give a better limiting value of $e$ than the $e_{\max }$ given by Eq. (18) in Weidenschilling and Jackson (1993).

In a three dimensional case, however, the condition for a stable trap may not exist, due to the effect of drag on the orbital inclination.

\section{NUMERICAL EXPERIMENTS}

\subsection{1:1 Mean Motion Resonance in the Coplanar Circular Sun-Jupiter-Dust Case}

In this section, we show the results from our numerical simulations. The equations of motion of the Sun, Jupiter, and a dust particle are integrated using RADAU (Everhart 1985) on a HP9000 715/75 workstation. The Sun and Jupi-

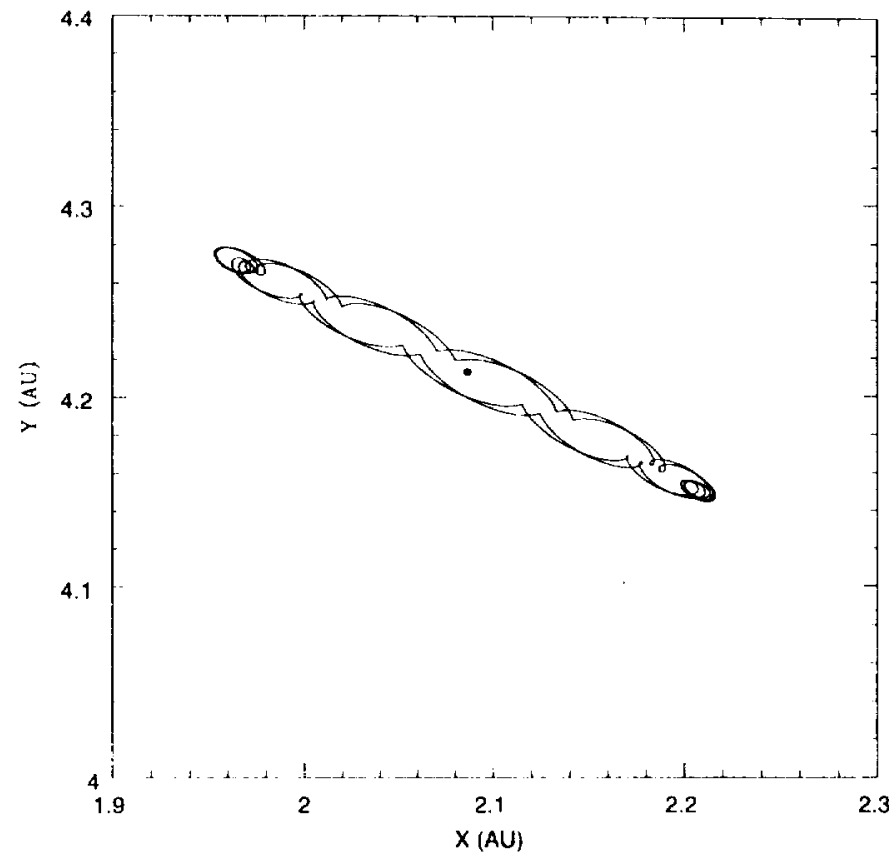

FIG. 4. Positions of the test particle, with $\beta=0.26$ and $\mathrm{sw}=0.35$ in the rotating reference frame for the first 300 years in the planar, circular Sun-Jupiter-dust system. The Lagrangian equilibrium point L4 is labeled by the black dot.

ter are gravitationally interacting in circular orbit with each other and the dust particle while the dust particle does not affect the motions of the Sun and Jupiter. Jupiter's semimajor axis is set to $5.2 \mathrm{AU}$. The eccentricity of the test particle is initially set to near zero to examine the circular orbit case. We use $\beta=0.26$ and $\mathrm{sw}=0.35$, corresponding to a $1-\mu \mathrm{m}$-radius dust particle of density $2.7 \mathrm{gm} \mathrm{cm}^{-3}$ trapped in the $1: 1$ mean motion resonance with Jupiter (Paper I).

The first test particle is initially placed very close to L4. It is trapped in the 1:1 mean motion resonance with Jupiter for several hundred thousand years before it has a close encounter with Jupiter at 250,651 years and is removed from the resonance. Initially the particle is in a tadpole orbit around L4 (Fig. 4). Its orbit expands gradually and becomes a horseshoe after 234,200 years (Fig. 5). The variation in $C$ over the particle's trapping life time is shown in Fig. 6. Note that this particle's orbit becomes a horseshoe when its Jacobi constant, $C$, exceeds $C_{\mathrm{L} 3}$ (Table I), as we expected from the analysis in Section 3. Although this causes the oscillatory amplitude of $\phi$ to suddenly increase, $\dot{\phi}$ and therefore $\dot{C}$ merely continue to smoothly increase with time.

In Fig. 7, we show the detailed variation of $C$ from 200,000 to 204,000 years, as an example to demonstrate Eq. (17). Qualitatively, it agrees perfectly with Eq. (17), with a large short-term variation and a small secular in- 


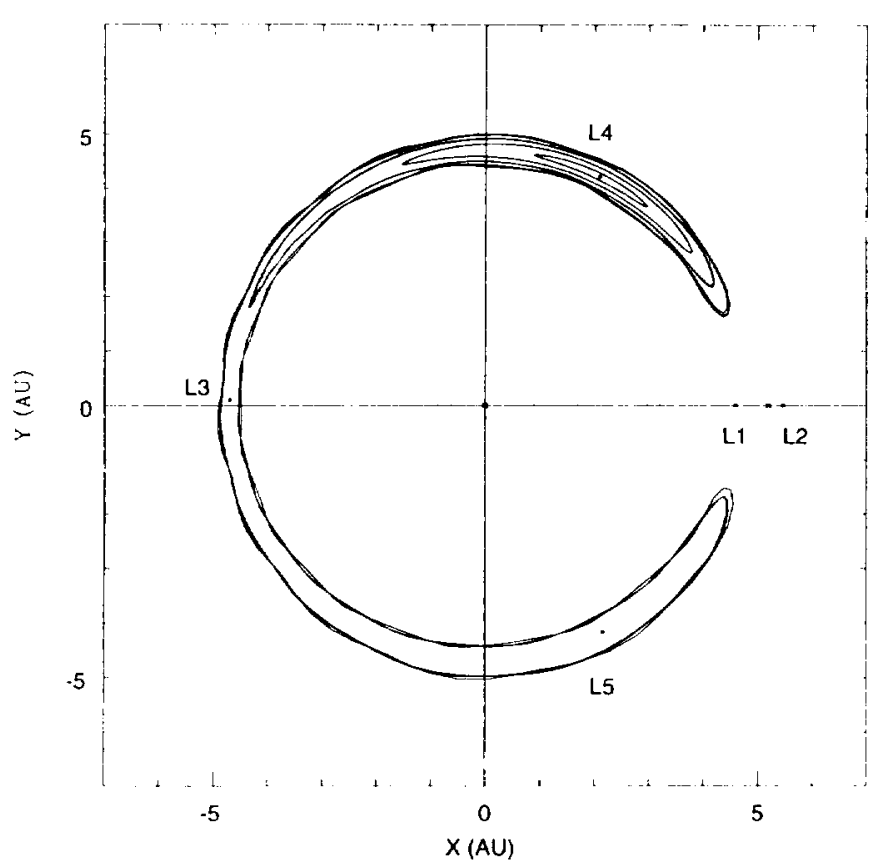

FIG. 5. Positions of the same test particle from Fig. 4, in the rotating reference frame at four different time periods. They are, from inside out, 140,000 to 141,000 years, 200,000 to 201,000 years, 230,000 to 231,000 years, and 246,000 to 247,000 years. We connect positions that are two years apart to form the curves. The five Lagrangian equilibrium points are indicated as black dots. The positions of Sun and Jupiter are labeled by crosses. crease. The time derivative of $C$ from Fig. 7 is also compared, quantitatively, with the right-hand side of Eq. (17). The comparison is in Fig. 8a. The curve is the time derivative of $C$ from Fig. 7 while the points are the results by using positions of the test particle as functions of time to calculate the right-hand side of Eq. (17). The agreement is excellent. Note the large oscillatory variation, due to $\dot{\phi}$, and the smaller positive secular increase with time, mostly due to $\dot{\phi}^{2}$ (Fig. 8b). Figure $8 \mathrm{c}$ shows the still smaller secular increase due to $(\dot{r} / r)^{2}$. These three figures show that Eq. (17) does describe the time variation of the Jacobi constant correctly.

If a test particle is placed initially near LS instead of L4, its evolution is similar to the above case. Based on our analysis in Section 3, there is no difference between $C_{\mathrm{LA}}$ and $C_{\mathrm{L} S}$ for particles with $\beta$ as large as 0.26 , and $\mathrm{sw}=0.35$, in the restricted Sun-Jupiter-dust system. Our numerical experiments also show no difference in terms of the global behavior between these two cases.

\subsection{I:I Mean Motion Resonance in the Nonplanar and Noncircular Sun-Jupiter-Dust Cases}

In this section, we show some numerical examples of the nonplanar, noncircular cases. The first one is a dust particle that starts with an eccentricity of 0.1 and the longitude of perihelion at $0^{\circ}$, moving in the Sun-Jupiter system. Jupiter is assumed to have a circular orbit. This is still a circular (circular Jupiter orbit) case, but the eccen-

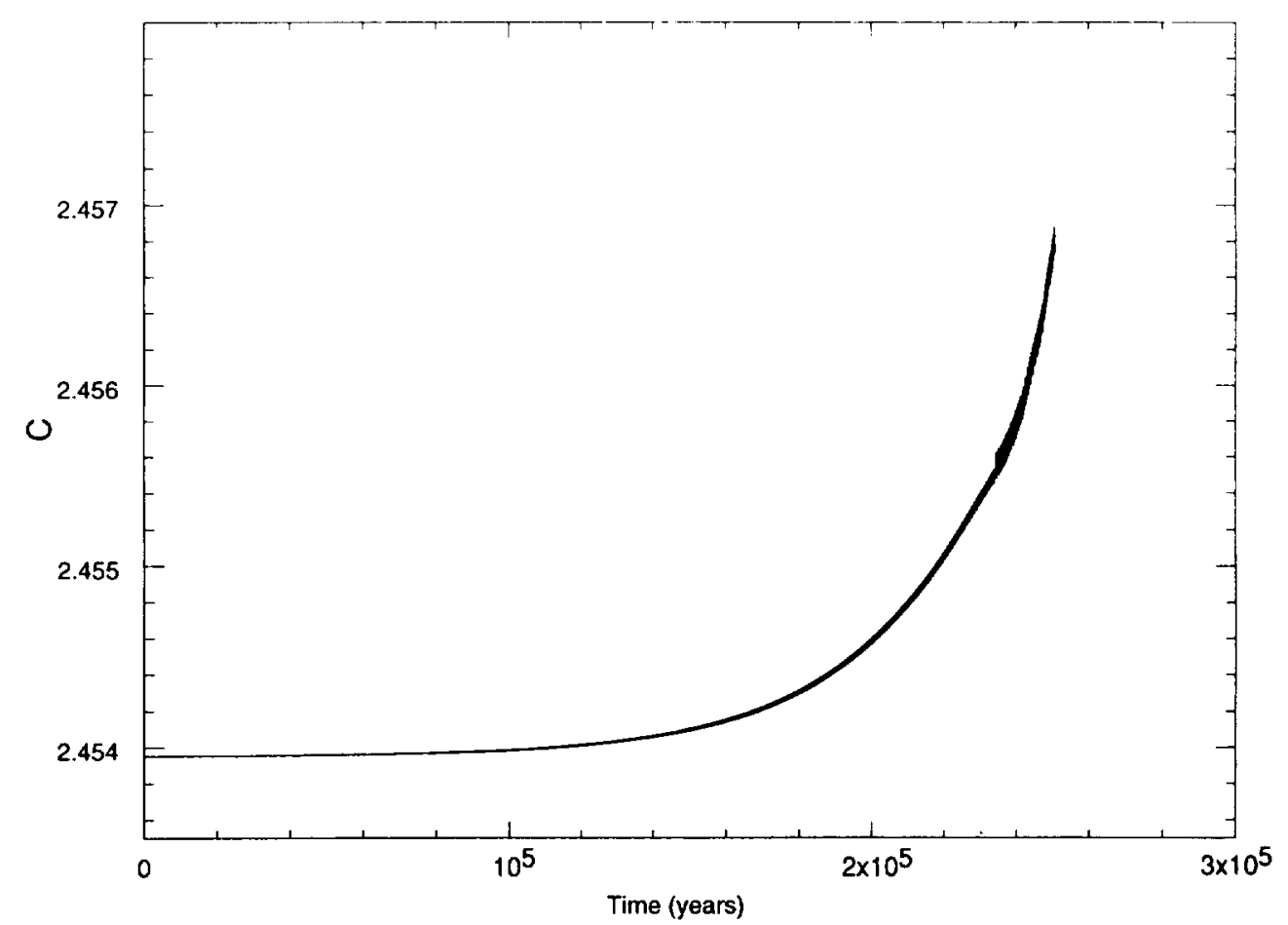

FIG. 6. The Jacobi constant of the test particle over a period of 250,651 years in the coplanar, circular restricted Sun-Jupiter-dust system. 


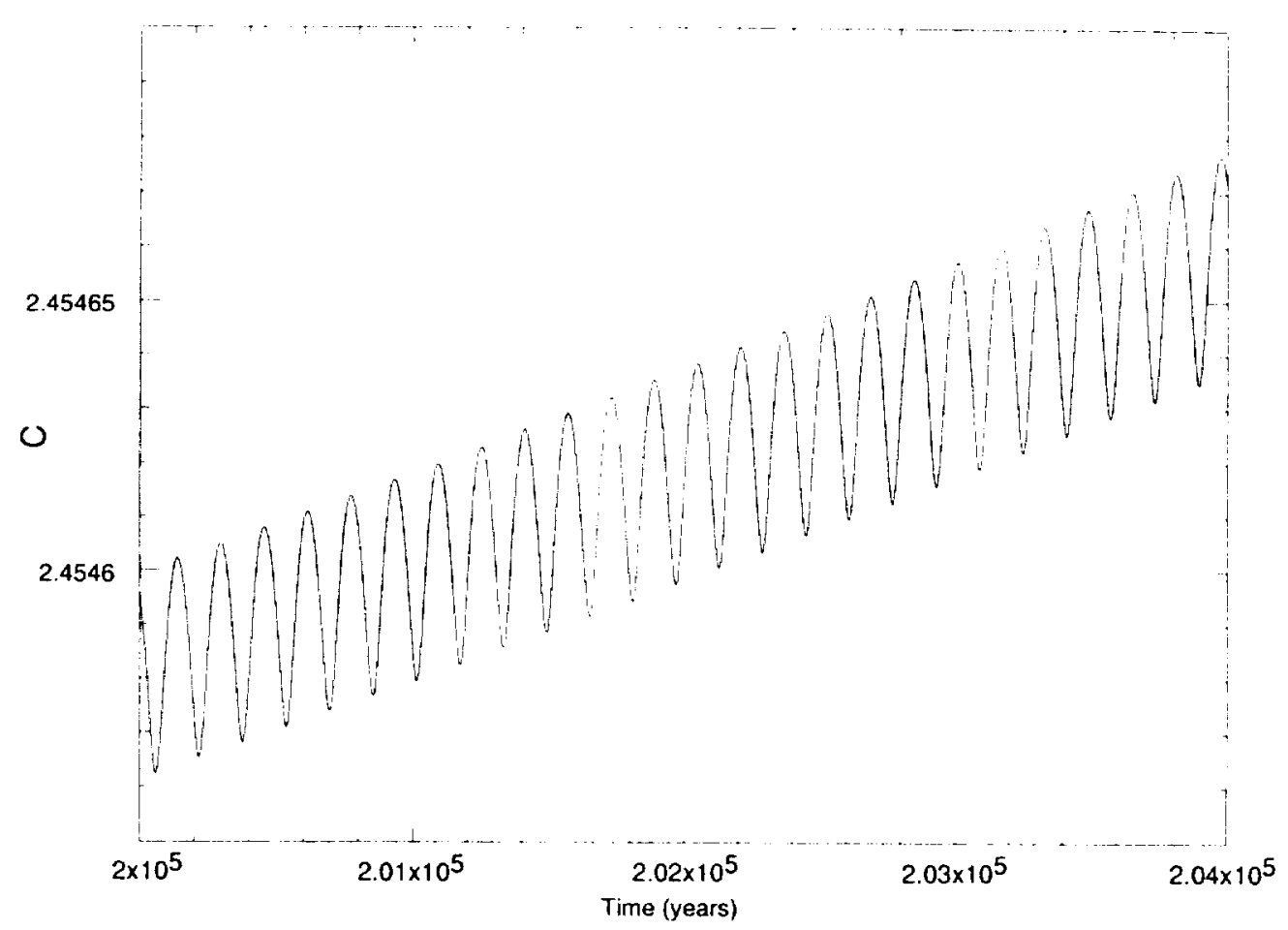

FIG. 7. A detailed plot of part of Fig. 6, from 200,000 to 204,000 years.

tricity of the dust particle, which decays with time, makes the overall evolution quite different from the previous case. The dust particle is initially placed near L4. It starts with a tadpole orbit and the orbit gradually expands and becomes a horseshoe at 193,660 years (Fig. 9). The horseshoe orbit keeps expanding until the particle has a close encounter with Jupiter at 204,476 years. The particle is then removed from the resonance. In Fig. 10 we show the variation of $C$ of the particle. Detailed examination of the curve shows that the particle's orbit becomes a horseshoe when its $C$ value exceeds $C_{\mathrm{L} 3}$. The short-term variation does not seem to be too much larger than that in the planar, circular case, while the secular increase is more dramatic, due to the particle's eccentricity effect on $\dot{r}$. The total trapping life time is significantly shorter than that of the circular case.

The next example is a dust particle with $5^{\circ}$ inclination with respect to Jupiter's orbital plane. The longitude of the ascending node is $30^{\circ}$. Both Jupiter and the dust particle have circular orbits. This dust particle starts its orbit as a tadpole around L4. Its orbit expands and becomes a horseshoe at 236,656 years. Finally the particle has a close encounter with Jupiter at 245,579 years and is removed from the resonance. The variation in $C$ of the particle is shown in Fig. 11. Qualitatively, its overall behavior is similar to the circular planar case with a large short-term variation; however the initial secular increase is faster with time because of the $\dot{\theta}^{2}$ components. This fast increase slows down as the dissipative nature of drag contracts the particle into the $(x, y)$ plane (i.e., reduces its orbital inclination) where the potential is a minimum along the $z$ direction. The $e$-folding time according to Eq. (26) is about 202,500 years, which is in good agreement with the decay in inclination from the numerical result (Fig. 12). There is no significant change in the total trapping life time for this inclination compared with the planar case.

A third example is a dust particle with eccentricity of 0.1 , longitude of perihelion at $70^{\circ}$ and inclination of $5^{\circ}$ (with respect to the orbital plane of Jupiter) and longitude of the ascending node at $30^{\circ}$. The eccentricity of Jupiter is 0.048 . This particle starts with a tadpole orbit around L4 and becomes a horseshoe at 223,600 years. It has a close encounter with Jupiter at 229,463 years and is removed from the resonance after that. The variation in $C$ of the particle is shown in Fig. 13. Both its long-term and short-term variations are much more complicated than the previous cases. However, it is still the secular increase component that gradually enlarges the forbidden regions that forces the orbit to expand from a tadpole to a horseshoe and to have a close encounter with Jupiter.

As we can see from the above examples, the evolution of an orbit becomes more complicated when the eccentricity and inclination of the dust grain are involved. Likewise when Jupiter's orbital eccentricity is considered. However, although the planar circular analysis in Section 3 


\section{a}
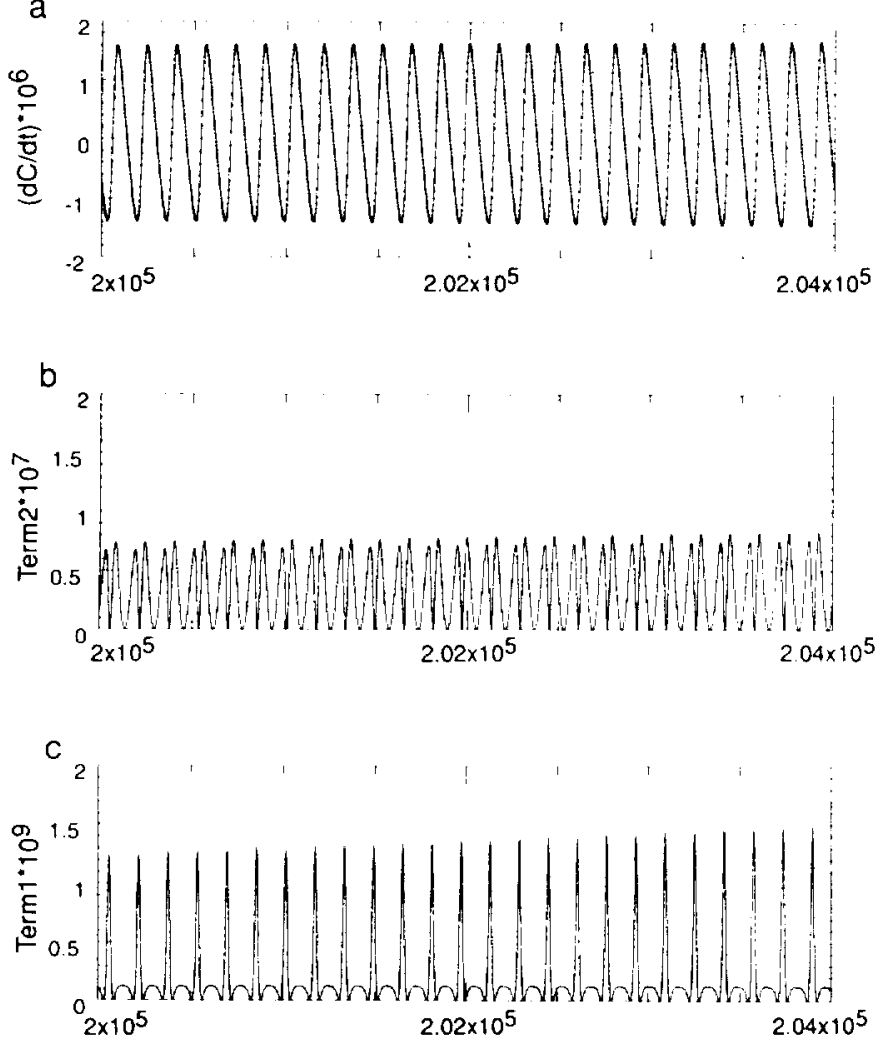

Time (years)

FIG. 8. (a) Comparison between the time derivative of $C$ from Fig. 7 (shown as curve) and the results (shown as black dots) by using positions of the dust particle as functions of time to calculate the righthand side of Eq. (17). The agreement is excellent. (b) The contribution of $\dot{\phi}^{2}$ term in the bracket of Eq. (17) to the secular increase of $\dot{C}$. Term $2=2(1+s w)\left(\beta \mu_{1} / c\right) \dot{\phi}^{2}$. (c) The contribution of $2(\dot{r} / r)^{2}$ term in the bracket of Eq. (17) to the secular increase of $\dot{C}$. Term $1=2(1+s w)\left(\beta \mu_{1} / c\right)[2(\dot{r}\}$ r) $\left.)^{2}\right]$.

does not describe in detail the evolution of an orbit in these cases (especially the last one), it still provides guidance in understanding the nature of the evolution. The natural trend for an orbit under the influence of PR and solar wind drag is to evolve from a tadpole to a horseshoe which eventually leads to a close encounter with Jupiter. An analytical method for the eccentric Jupiter system with mutual inclination between objects may be possible to develop, but much can be learned from direct numerical simulation alone. The basic physics involved is the same as in the case of the planar circular system.

\subsection{Implication to Asteroidal Dust Particles and \\ Trojan Dust Particles Trapped in the 1:1 Mean Motion Resonance with Jupiter}

The trapped ring particles we predicted to exist from our numerical analyses in Paper I were of main belt asteroidal origin; they had $\beta=0.26$ and $\mathrm{sw}=0.35$ and were often injected right into the $1: 1$ mean motion resonance location (which, with this value of $\beta$, is somewhat interior to Jupiter's orbit) when they were released from their asteroidal parent bodies. In addition to Jupiter, there were six other planets perturbing their orbits (in our numerical simulation, we did not include Mercury and Pluto). The initial eccentricities of the trapped dust grains lay between 0.1 and 0.35 while their typical inclinations lay between $0^{\circ}$ and $20^{\circ}$. Their typical initial libration angles (angle from Jupiter) were about $45^{\circ}$ to $135^{\circ}$. While they were trapped, their orbits expanded from tadpole orbits to horseshoe orbits. Most of the particles got out of the resonance within 30,000 years. Close encounters with Jupiter caused them to be removed from the resonance (Paper I). Dust particles, with considerably smaller $\beta$ values, produced from Trojan asteroids can also be trapped in the $1: 1$ resonance (Paper 1). Their orbital evolution is similar to that of the asteroidal dust particles. As a matter of fact, any dust particles trapped in a $1: 1$ resonance with any planet will behave similarly. Their evolution can all be described by the analyses in Section 3.

The real Solar System is more complicated than a simple circular restricted Sun-Jupiter-dust system. But the physical nature of the evolution of those dust particles is

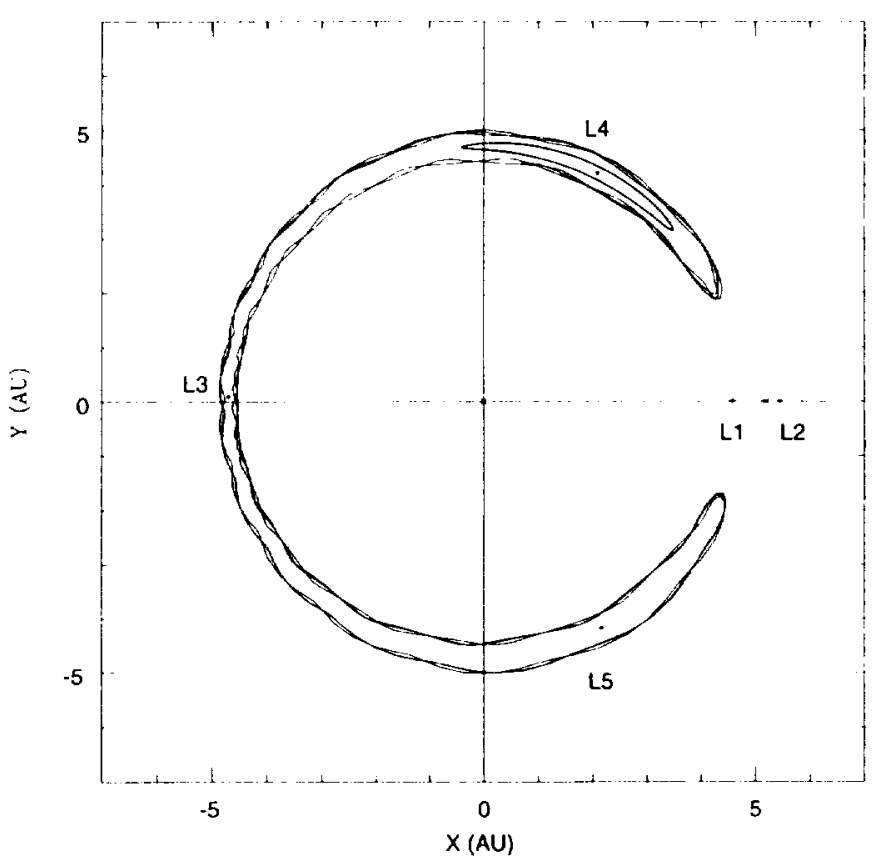

FIG. 9. Positions of a test particle that started with 0.1 eccentricity and $0^{\circ}$ longitude of perihelion in the rotating reference frame at two different time periods. The Sun and Jupiter are in circular orbits and the dust particle orbits in the same plane. The inner curve is from 140,000 to 141,000 years (the dust particle's eccentricity has dropped to less than 0.01 ). The outer curve is from 200,000 to 201,000 years. Again, we connect points that are two years apart to form the curves. 


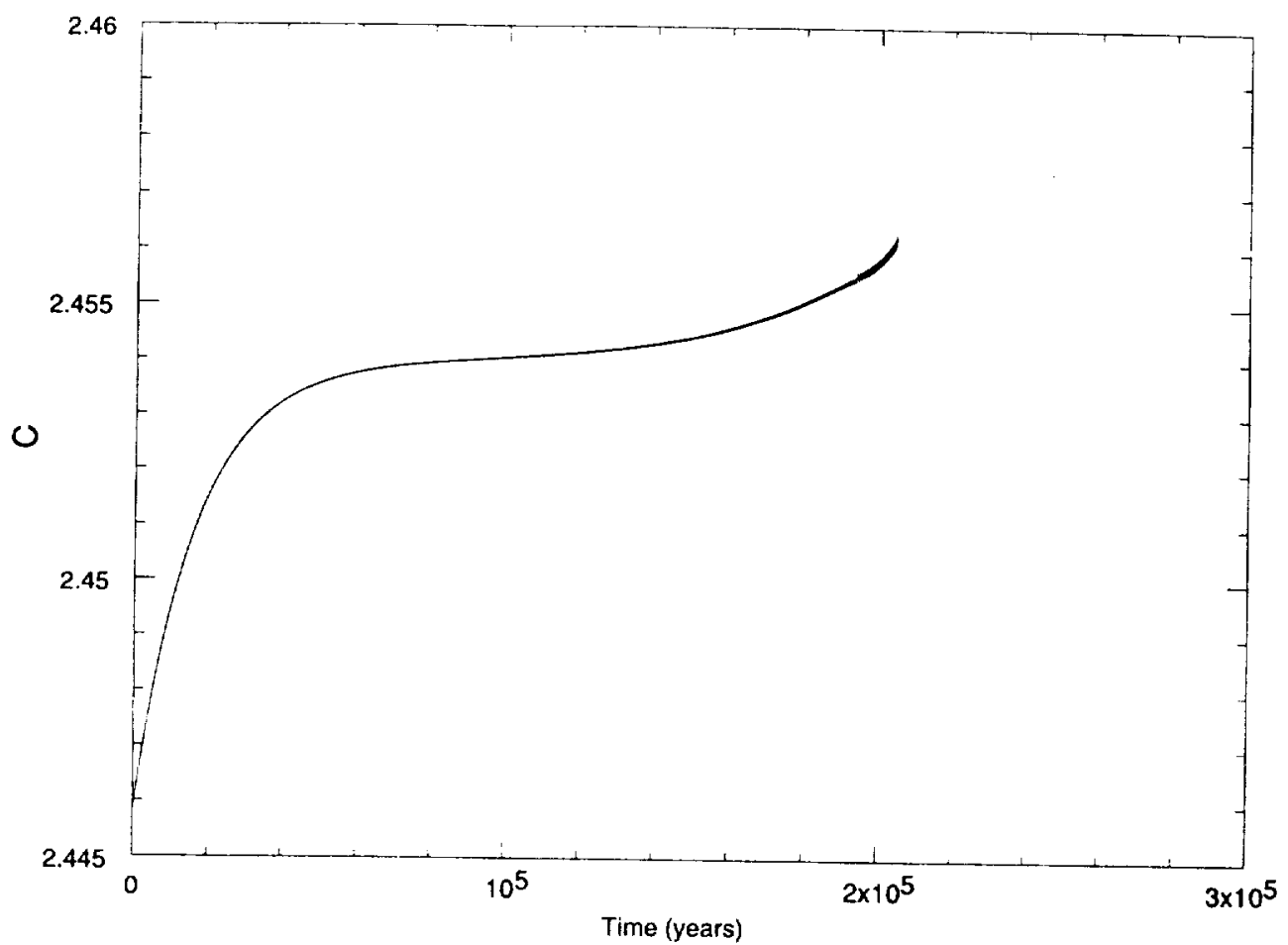

FIG. 10. The Jacobi constant of the test particle from Fig. 9 over the period of 204,476 years. The particle's orbit becomes a horseshoe when $C$ exceeds $C_{13}$.

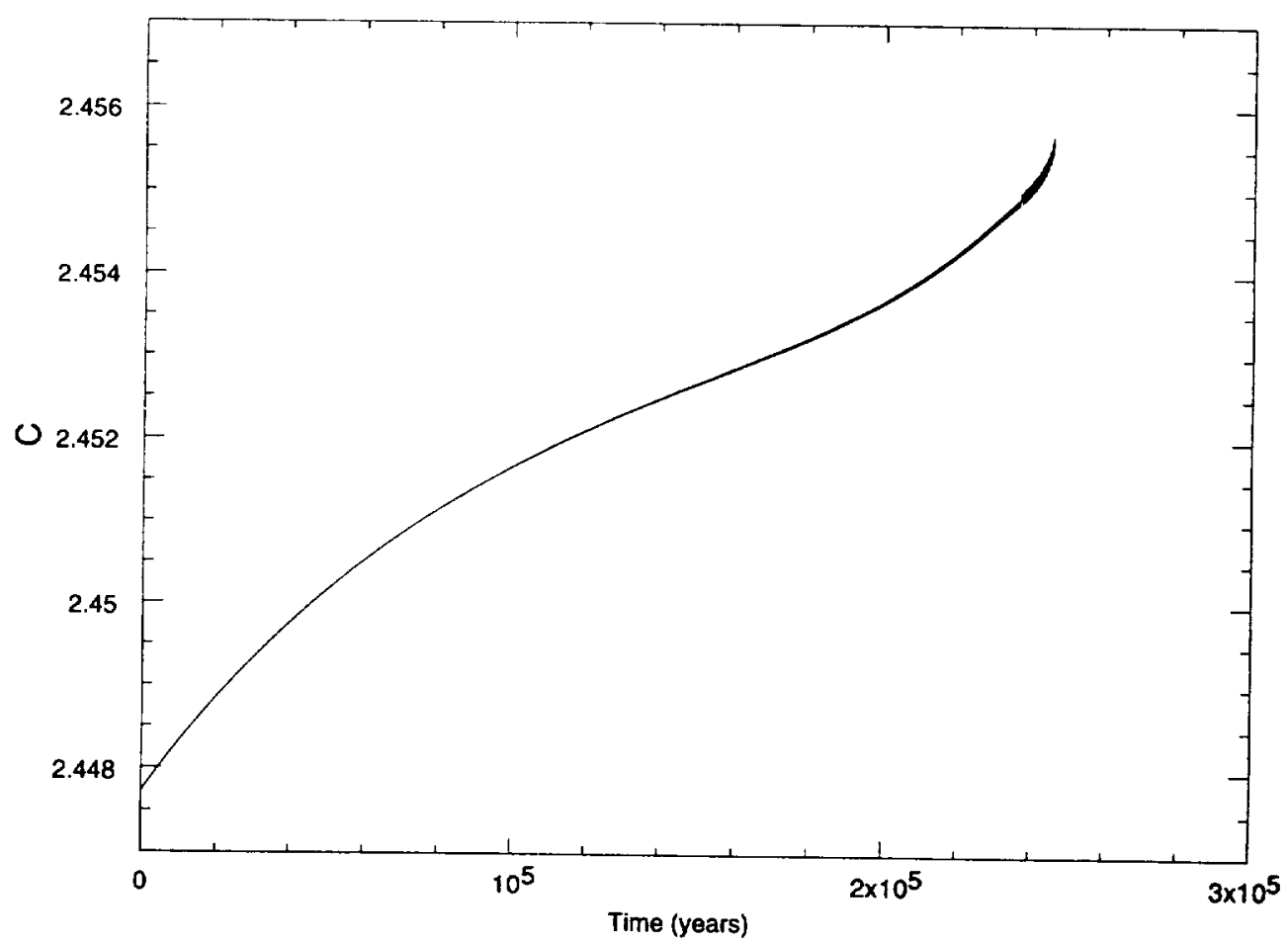

FIG. 11. The Jacobi constant of a test particle over the period of 245,579 years. The test particle has an initial inclination of $5^{\circ}$ (with respect to Jupiter's orbital plane) and longitude of the ascending node at $30^{\circ}$ in a circular Sun-Jupiter-dust system. 


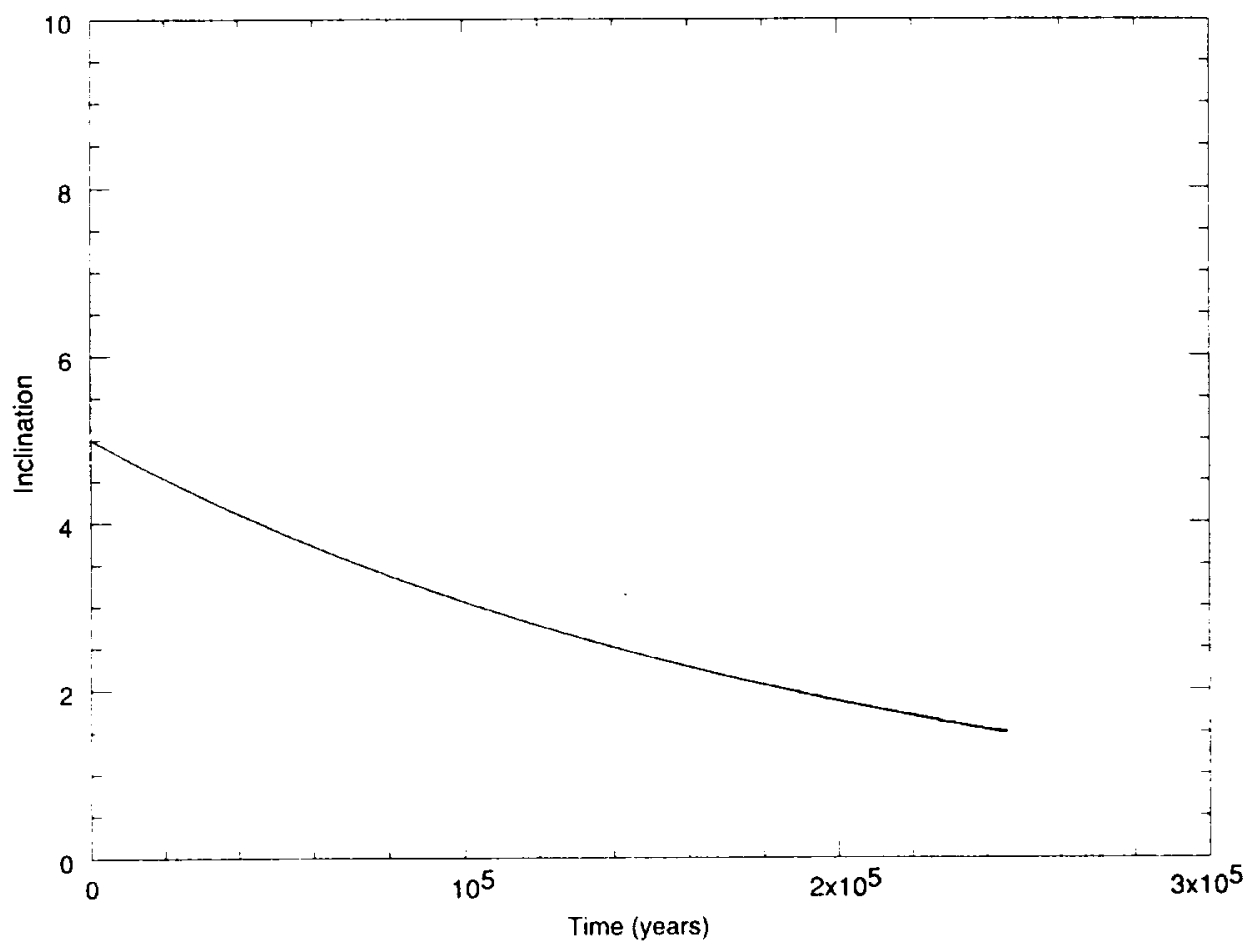

FIG. 12. The decay of orbital inclination of the test particle in Fig. 11. The predicted $e$-folding time from Eq. (26) is about 202,500 years.

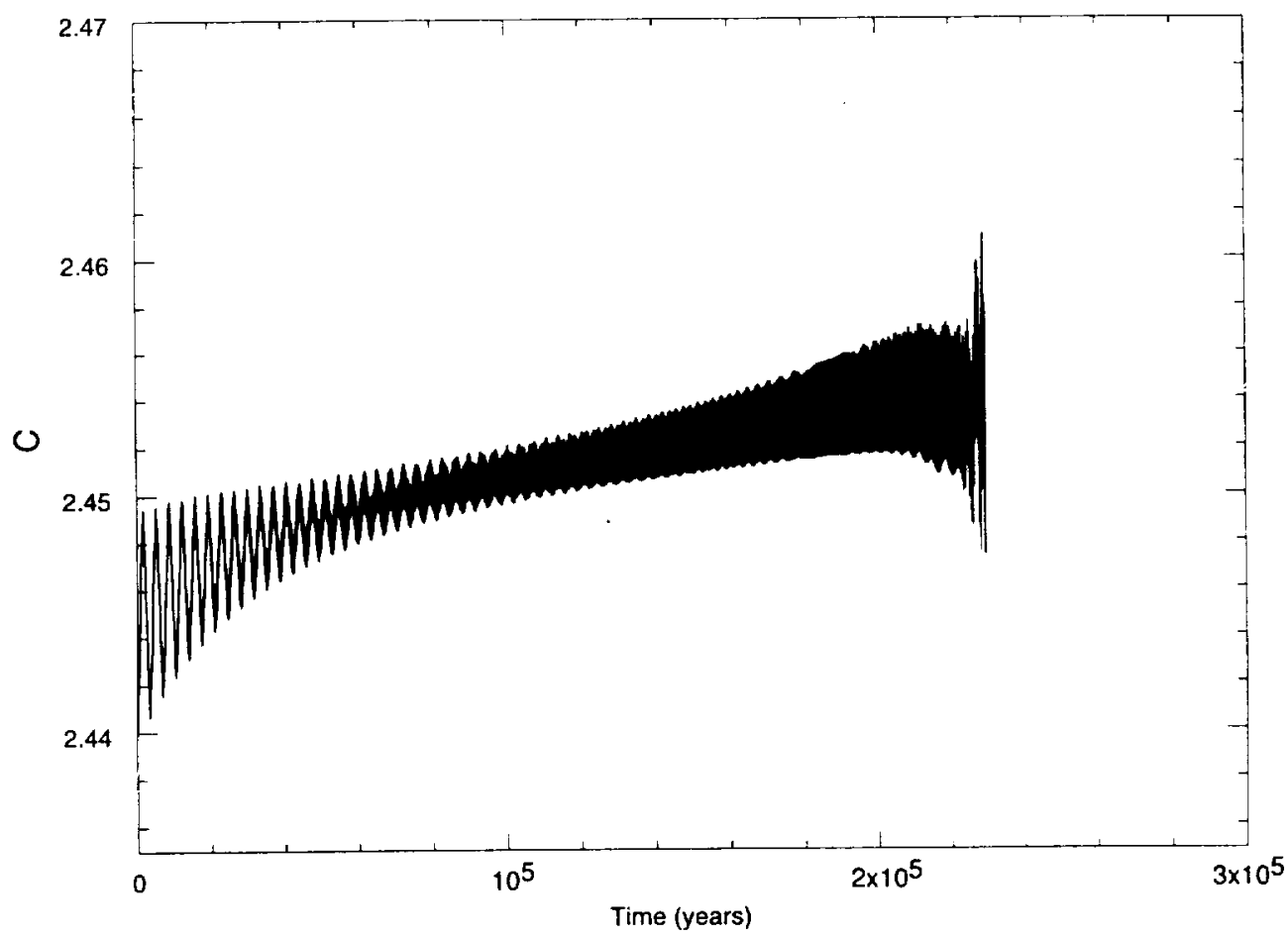

FIG. 13. The Jacobi constant of a test particle over the period of 229,463 years. The test particle initially has an inclination of $5^{\circ}$ (with respect to Jupiter's orbital plane) with longitude of the ascending node at $30^{\circ}$ and an eccentricity of 0.1 with longitude of perihelion at $70^{\circ}$. The eccentricity of Jupiter is 0.048 . Its longitude of perihelion is set to $0^{\circ}$. 
still qualitatively described by our analysis. When drag forces are negligible, particles can get trapped for a much longer time scale, as in the case of the present Trojan asteroids. When PR and solar wind drag are included, the Jacobi constant of a dust particle will increase as is shown by Eq. (16). This leads to the expansion of an orbit from a tadpole to a horseshoe and, eventually, a close encounter with Jupiter becomes unavoidable. This is in good agreement with the results from Paper I.

We can also compare the trapping life time of dust particles in Paper I with our analysis in this paper. In the planar, circular Sun-Jupiter-dust system, if a dust particle has $\beta=0.26$, sw $=0.35$ and starts with a libration angle from $45^{\circ}$ to $135^{\circ}$ (similar to the second inner most orbit in Fig. 5), it will remain trapped for approximately 50,000 years before having a close encounter with Jupiter (use the data from Section 4.1). If we consider the effects of eccentricity ( 0.048 for Jupiter and up to 0.35 for dust particles) and inclination (up to $20^{\circ}$ ) on the system, they have the ability to speed up the growth of an orbit through increased $\dot{\theta}^{2}$ and $(\dot{r} / r)^{2}$ in Eq. (16); with the additional perturbation from other planets, 30,000 years seems to be a reasonable trapping life time for real asteroidal dust particles trapped in the 1:1 mean motion resonance with Jupiter.

Another phenomenon about the $1: 1$ trap, from our numerical calculations in Paper $I$, is that it is impossible for a dust grain to drift toward and get trapped in the $1: 1$ resonance with Jupiter. This can easily be understood from the analyses in Section 3. When a dust grain is approaching, under PR and solar wind drag, from outside the location of $1: 1$ resonance toward it, the $C$ of the dust grain decreases. When $C$ reaches $C_{\mathrm{L} 1}$, trapping in the $1: 1$ resonance becomes possible. However, if the dust grain does get trapped, its $C$ immediately starts to increase (Eq. (16)) and expels the dust particle from the 1:1 resonance. Thus, it is impossible for a dust grain to drift toward and get trapped in a 1:1 resonance. Actually, when the planet involved is a giant planet, such as Jupiter, gravitational scattering usually prevents the dust grain from even reaching $C_{\mathrm{L} 1}$.

\section{CONCLUDING REMARKS}

Instead of the Jacobi constant, $C$, one can consider the "modified total energy" in the rotating reference frame defined by

$$
E_{\mathrm{J}}=-\frac{C}{2}=\frac{1}{2} V^{2}-U,
$$

where

$$
-U=-\frac{(1-\beta) \mu_{1}}{r_{1}}-\frac{\mu_{2}}{r_{2}}-\frac{1}{2}\left(x^{2}+y^{2}\right)
$$

is the Jacobi potential. Without drag, $E_{\mathrm{J}}$ is also a constant of the motion with a modified potential, $-U$, due to the rotation of the coordinate system (see, e.g., Danby 1989). It is also equal to the Hamiltonian constant in the rotating system (Szebehely 1967). This constant comes from the only explicitly determined integral of motion in the three-body system. Because of its close resemblance to the energy in the inertial system, it may be more appropriate to call $E_{\mathrm{J}}$ the Jacobi energy.

In the two-body Sun-dust problem the effect of the drag forces is to take away orbital energy and angular momentum from the particle (Wyatt and Whipple 1950, Burns et al. 1979). In the three-body rotating reference frame, drag acts to take away the modified energy, or Jacobi energy, of the particle while the grain is in a $1: 1$ trap. By following the variation of the Jacobi energy with time, we can gain some understanding of the physical nature of how an orbit evolves.

If there were no drag forces, $E_{\mathrm{J}}$ like $C$ would remain constant with time. At those points where $E_{\mathrm{J}}=-U$, the velocity is zero and we can think of the particle as pressed up against the side of the potential energy "hill." Coriolis forces (e.g., Greenberg and Davis 1978) then constrain the particle motion to librate around the hill-which may be either tadpole-shaped or horseshoe-shaped-leading to tadpole- or horseshoe-shaped orbits.

The PR and solar wind drag forces cause $E_{\mathrm{J}}$ to decrease with time (equivalent to the increase of $C$ ). This, in turn, forces the particle farther down the side of the potential hill and increases the region of exclusion (i.e., the total energy, $E_{\mathrm{J}}$, must always be greater than or equal to the potential energy, $-U$ ). Thus $\mathrm{PR}$ and solar wind drag forces cause the corresponding tadpole or horseshoe orbits to expand with time because dust particles must orbit at lower energies about an ever increasing circumference of the inaccessible part of the potential hill.

Relating the circular restricted case to the actual case of the Sun-Jupiter-dust system, where Jupiter is in an elliptical orbit about the Sun and gravitational perturbations from other planets must be considered, is still not straightforward, however, and we must rely on numerical modeling. But we believe the insights that we have gained with our new mathematical formulation helps in gaining a physical intuition for the cause of the ever increasing libration amplitudes.

\section{ACKNOWLEDGMENTS}

We thank C. D. Murray for sending us a preprint of his paper. Thanks also to R. Malhotra, C. D. Murray, and S. J. Weidenschilling for their many constructive review comments. This research was performed while J.C.L. held a National Research Council-NASA/JSC Research Associateship. 
Note added in proof. Since acceptance of our present paper for publication, we have become aware of a very interesting paper by Beaugé and Ferraz-Mello (Icarus 110, 239-260, 1994). They use a Hamiltonian treatment of the variation of resonance variables to derive their Eq. (15), and corresponding Table 1, which give relationships between orbital eccentricity and integer resonance ratios identical to the requirement we derive in our Eq. (33) for potentially stable trapping in exterior resonances. Our treatment differs from theirs in that it depends on an examination of the time variation of the Jacobi "constant."

\section{REFERENCES}

Burns, J. A., P. L. LAmy, AND S. Soter, 1979. Radiation forces on small particles in the solar system. Icarus 40, 1-48.

Chernikov, Y. A. 1970 . The photogravitational restricted three-body problem. Sou. Astron. 14, 176-181.

Colombo, G., D. A. Lautman, and I. I. Shapiro, 1966. The Earth's dust belt: Fact or fiction? 2. Gravitational focusing and Jacobi capture. J. Geophys. Res. 71, 5705-5717.

DANBY, J. M. A. 1989. Fundamentals of Celestial Mechanics, second ed., Willmann-Bell, Richmond, VA.

EvERHART, E. 1985. An efficient integrator that uses Gauss-Radau spacings. In Dynamics of Comets: Their Origin and Evolution (A. Carusi and G. B. Valsecchi, Eds.), pp. 185-202. Reidel, Boston.

GREENBERG, R. 1978. Orbital resonance in a dissipative medium. Icarus $33,62-73$.

GREENBERG, R., AND D. R. DAVIS, 1978. Stability at potential maxima: The $L A$ and $L 5$ points of the restricted three-body problem. Am. J. Phys. 46, 1068-1070.

Gustafson, B. A. S. 1994. Physics of zodiacal cloud. Ann. Rev. Earth Planet. Sci. 22, 553-595.

Henrard, J., AND A. Lemaitre, 1983. A second fundamental model for resonance. Celest. Mech. 30, 197-218.
JACKSON, A. A., AND H. A. ZoOK, 1992. Orbital evolution of dust particles from comets and asteroids. Icarus $97,70-84$.

Liou, J. C. 1993. Dynamical Evolution of Asteroidal and Cometary Particles and Their Contribution to the Zodiacal Cloud. Ph.D. thesis, University of Florida.

Liou, J. C., AND H. A. Zook, 1995. An asteroidal dust ring of micronsized particles trapped in the $1: 1$ mean motion resonance with Jupiter. Icarus 113, 403-414.

Marchal, C. 1990. The Three-body Problem. Elsevier, Amsterdam.

MARION, J. B. 1970. Classical Dynamics of Particles and Systems, second ed. Academic Press, New York.

MARZARI, F., AND V. VANZANI, V. 1994. Dynamical evolution of interplanetary dust particles. Astron. Astrophys. 283, 275-286.

Moulton, F. R. 1914. An Introduction to Celestial Mechanics, second ed. Dover, New York.

MURRaY, C. D. 1994. The dynamical effects of drag in the circular restricted three-body problem: I. The location and stability of the Lagrangian equilibrium points. Icarus 112, 465-484.

Peale, S. J. 1986. Orbital resonances, unusual configurations and exotic rotation states among planetary satellites. In Satellites (J. A. Burns and M. Matthews, Eds.), pp. 159-223. Univ. of Arizona Press, Tucson.

Peale, S. J. 1993. The effect of the nebula on the Trojan precursors. Icarus 106, 308-322.

SChuerman, D. W. 1980 . The restricted three-body problem including radiation pressure. Astrophys. J. 238, 337-342.

Szebehely, V. 1967. Theory of Orbits. Academic Press, New York.

WeIDENSChILling, S. J., AND A. A. JACKSON, 1993. Orbital resonances and Poynting-Robertson drag. Icarus 104, 244-254.

Wyatt, S. P., AND F. L. Whipple, 1950. The Poynting-Robertson effect on meteor orbits. Astrophys. J. 111, 134-141.

YODER, C. F. 1979. Notes on the origin of the Trojan asteroids. Icarus 40, 341-344. 


\section{$\checkmark$}

,

1

1 